\title{
Rumiación cognitiva y su relación con las psicopatologías internalizantes: una revisión sistemática
}

\author{
Cognitive rumination and its relationship whit the internalizing \\ psychopathologies: A Systematic Review
}

\author{
Ronald Alberto Toro \\ Maria Vulbuena-Martin \\ Danna Riveros-Lopez \\ Maria Zapata-Orjuela \\ Andrea Florez-Tovar \\ Universidad Católica de Colombia
}

\begin{abstract}
Resumen
La rumiación cognitiva $(\mathrm{RC})$, se considera un estilo de respuesta cognitiva repetitiva y pasivamente focalizada ante el malestar, que se ha identificado en los síntomas psicopatológicos internalizantes como la ansiedad, depresión y la somatización. El objetivo del estudio fue identificar mediante una revisión sistemática los aportes investigativos entre los años 2010 y 2019 sobre la relación entre la rumiación cognitiva y la psicopatología internalizante, basados en los lineamientos del modelo PRISMA en los idiomas inglés y español, en las bases de datos científicas como Science Direct, Psicodoc, Proquest, PsyArticles y Medline, en las que se tuvo en cuenta como descriptores principales la rumiación cognitiva y la psicopatología internalizante. Se recolectaron 11730 artículos de los cuales se evaluaron 103 artículos de texto completo para elegibilidad, con 31 artículos finales incluidos en la revisión sistemática. Se encontró que la $\mathrm{RC}$ es un predictor clave de los trastornos de ansiedad y depresión, mientras que en somatización no hay evidencia concluyente, únicamente en el dolor crónico. Además, se identificó que la RC se presenta más en mujeres y en todas las edades. A nivel explicativo, se asocia a estilos cognitivos disfuncionales como factores de vulnerabilidad y mantenimiento de la psicopatología internalizante.

Palabras clave: revisión sistemática, rumiación cognitiva, ansiedad, depresión, somatización

\footnotetext{
Nota del autor

Ronald Alberto Toro Departamento de Psicología, Universidad Católica de Colombia. Maria Vulbuena-Martin Departamento de Psicología, Universidad Católica de Colombia. Danna Riveros-Lopez Departamento de Psicología, Universidad Católica de Colombia. Maria Zapata-Orjuela Departamento de Psicología, Universidad Católica de Colombia. Andrea Florez-Tovar Departamento de Psicología, Universidad Católica de Colombia. La correspondencia en relación con este artículo debe dirigirse a Ronald Alberto Toro, Departamento de Psicología, Universidad Católica de Colombia, Jardín Juárez \#147, Centro Histórico C.P. 98000 , Zacatecas, Zacatecas, México. Dirección electrónica: ratoro@ucatolica.edu.com
} 


\begin{abstract}
Cognitive Rumination (CR), is a cognitive response style to discomfort, repetitive and passively focused on internalizing psychopathological symptoms such as anxiety, depression, and somatization. The aim was to identify through a systematic review of the research contributions between 2010 and 2019 on the relationship between cognitive rumination and internalizing psychopathology. It was based on the PRISMA model, analyzing the scientific databases such as Science Direct, Psicodoc, Proquest, PsyArticles and Medline, in which it was considered as the main descriptors the cognitive rumination and internalizing psychopathology. 11730 articles were collected which 103 full-text articles were evaluated for eligibility, with 31 final articles included in the systematic review. It was found that $\mathrm{CR}$ is a key predictor for anxiety and depression disorders, while in somatization there is no conclusive evidence, only in chronic pain. It was also found that CR occurs more in women at all ages. At an explanatory level, it is associated with dysfunctional cognitive styles as factors of vulnerability and maintenance of internalizing psychopathology.
\end{abstract}

Keywords: Systematic review, cognitive rumination, anxiety, depression, somatization

La rumiación cognitiva ( $\mathrm{RC}$ ), se define como un estilo de respuesta cognitiva repetitivo $\mathrm{y}$ pasivamente focalizado ante el malestar emocional, en los síntomas psicopatológicos y en las posibles causas y consecuencias de estas manifestaciones, asociadas a una escasa solución activa de problemas, aunque la persona refiera tenerlos enfocados y reconocer su malestar emocional que le acompaña permanentemente (Nolen-Hoeksema et al., 2008). Consiste en una secuencia de pensamientos repetitivos que se caracterizan por generar afectaciones perdurables, estáticas, incontrolables y cíclicas, evidentes en estados emocionales aversivos como el nerviosismo, miedo, disgusto, culpa o ira, derivados de procesos cognitivos recurrentes y no intencionales que surgen durante y después de un episodio percibido como negativo (Alcázar-Olán et al., 2018; Nolen-Hoeksema et al., 2008).
La RC se considera un constructo bidimensional, la primera dimensión es la reflexión, definida como un proceso introspectivo que tiene como propósito participar en la solución de problemas cognitivos; la segunda es la rumiación negativista, se caracteriza por la comparación constante ante situaciones actuales con estándares inalcanzables (González et al., 2017). La RC reflexiva se considera un constructo funcional que le permite al individuo solucionar problemas y aliviar estados de ánimo bajos, mientras que la $\mathrm{RC}$ negativista es una estrategia disfuncional acompañada de afecto negativo, la cual puede ser considerada como un factor de vulnerabilidad para la depresión y los trastornos de ansiedad (Grant y Beck, 2010).

Además, se ha planteado que la RC como medida global tiene efectos negativos a largo plazo como el incremento de los pensamientos negativos, la interrupción del procesamiento 
para la resolución de problemas, la interferencia en el afrontamiento activo, el mantenimiento de estresores negativos y la disminución del círculo de apoyo social (Lo et al., 2010). De esta manera, la RC al presentarse de manera repetitiva en el pensamiento puede estar asociada a la aparición e incremento de miedos específicos, mayor sintomatología depresiva, disminución de la autoestima, problemas en las relaciones sociales, trastornos de conducta y dificultad en los procesos de aprendizaje (Epkins et al., 2013).

La psicopatología internalizante está estrechamente ligada a la RC (Nolen-Hoeksema et al., 2008). Las problemáticas externalizantes como la depresión, ansiedad, y somatización, hacen referencia a los trastornos en los que se desarrolla, mantiene, experimenta y exhibe una angustia silenciosa e interna (Achenbach et al., 2016). Esta categoría describe los comportamientos que tienen una función hacia sí mismo, afectan al sujeto en los procesos individuales de interacción dentro del medio que se desenvuelve, se asocia a somatizaciones, rasgos de personalidad ansiosos y dependientes, procesos de aprendizaje desadaptativos como fobias y miedos, inestabilidad emocional y dificultad en la resolución de problemas recurrentes en el pensamiento, y trastornos depresivos y ansiosos (Grant y Beck, 2010).

Estas psicopatologías presentan un mayor predominio en la población, según la Organización Mundial de la Salud (OMS, 2017), los trastornos por depresión y ansiedad son problemas habituales de salud mental que afectan a la capacidad de trabajo y la productividad. Más de 300 millones de personas en el mundo sufren depresión, un trastorno que es la principal causa de discapacidad, y más de 260 millones tienen trastornos de ansiedad (Gaviria et al., 2017).

Actualmente, desde la perspectiva transdiagnóstica en la psicología clínica, se han generado avances en las investigaciones que aportan a la comprensión de las causas del comportamiento humano desadaptativo. En revisiones sistemáticas y metanálisis se ha reportado la reducción del pensamiento repetitivo mediante técnicas de mindfulness y técnicas cognitivo conductuales (Querstret y Cropley, 2013), también en otras revisiones se ha reportado como factor proximal de la desesperanza y suicidio (Morrison y O'Connor, 2008), y depresión postnatal, en los que se han identificado déficits en los mecanismos cognitivos como la rumiación, los sesgos, y el escaso control (DeJong et al., 2016). Estos hallazgos permiten inferir que los avances en torno a la rumiación como variable de interés en la psicopatología han tenido un creciente interés, sin embargo, no se cuenta con reportes recientes con un mayor alcance explicativo.

En una exploración de los reportes enfocados hacia el papel de la RC en la psicopatología internalizante, se identificó que desde el año 2010 no se dispone de una revisión sistemática ni metanálisis que den cuenta de los avances en las publicaciones científicas; dada 
la conexión entre los procesos cognoscitivos disfuncionales y el mantenimiento de las diferentes psicopatologías reportadas, esto permitía una mayor comprensión del papel mediador de las variables transdiagnósticas conocidas en la actualidad (Belloch, 2012). Por lo tanto, el objetivo fue identificar mediante una revisión sistemática los aportes investigativos entre los años 2010 y 2019 sobre la relación entre la rumiación cognitiva y la psicopatología internalizante, basados en los ítems del modelo PRISMA [Preferred Reporting Items for Systematic Reviews and Meta-Analyses] (Moher et al., 2009).

\section{Método}

\section{Protocolo y registro}

Para la revisión de los artículos seleccionados se creó una matriz en el programa Excel, en el que se registraron las citas bibliográficas de cada uno de los artículos extraídas del programa Mendeley 8.1. El proceso de revisión de los artículos se realizó por diferentes filtros, con los que se analizaron con base en la pertinencia acorde a los criterios de exclusión.

\section{Criterios de elegibilidad}

Se delimitaron los criterios de exclusión, los cuales se basaron en artículos que incluyeran temáticas como consumo de sustancias psicoactivas, factores biológicos, aplicación de tratamiento, trastornos mentales, comprobaciones teóricas, estudio de caso, revisión teórica, procesos cognitivos, pensamiento negativo, victimización, ideación suicida, relaciones de pareja, vulnerabilidad cognitiva, validación de pruebas psicométricas, enfermedad física, procesos sociales y estrategias de regulación emocional.

\section{Fuentes de información}

Se realizó una búsqueda sistemática entre el 9 de agosto y el 5 de noviembre del año 2018. La búsqueda bibliográfica se efectuó en las bases de datos electrónicas Medline, SciELO, Psicodoc, Proquest y Science Direct, con los siguientes términos descriptores: rumination and internalizing, rumination and positive affect and negative affect and anxiety, cognitive rumination, en los idiomas inglés y español, teniendo en cuenta las fechas de los estudios desde el 2010 hasta 2019 (Tabla 1).

\section{Búsqueda}

Por medio de la búsqueda electrónica, en las bases de datos reportadas en la Tabla 1, se ingresaron los descriptores y se filtró por los años de referencia (2010-2019), tipo de artículo y título de publicación, se procedió a descargar los artículos en formato ris y se exportó al programa Mendeley 8.1. En este último software, se eliminaron los duplicados y se actualizó el listado que incluye los elementos descriptivos del artículo como la referencia tipo APA, el abstract y los demás metadatos del documento elegido. 


\section{Tabla 1}

Descriptores de búsqueda

\begin{tabular}{|c|c|c|c|}
\hline Descriptores & Total & Fuente & Año \\
\hline Rumination and internalizing & 875 & Science Direct & 2010-2019 \\
\hline $\begin{array}{l}\text { Rumination and positive affect and negative affect } \\
\text { and depression }\end{array}$ & 3048 & Science Direct & 2010-2019 \\
\hline $\begin{array}{l}\text { Rumination and positive affect and negative affect } \\
\text { and anxiety }\end{array}$ & 2800 & Science Direct & 2010-2019 \\
\hline Rumination and depression & 327 & Medline & 2010-2019 \\
\hline Rumination and anxiety & 182 & Medline & 2010-2018 \\
\hline Rumination and depression & 92 & Psyarticles & 2010-2019 \\
\hline Rumination and anxiety & 59 & Psyarticles & 2010-2018 \\
\hline Rumination and depression & 4347 & $\begin{array}{l}\text { Proquest (Psy- } \\
\text { chology database } \\
\text { y Health \& Medical } \\
\text { Collection) }\end{array}$ & $2010-2018$ \\
\hline Rumination and anxiety & 4240 & $\begin{array}{l}\text { Proquest (Psy- } \\
\text { chology database } \\
\text { y Health \& Medical } \\
\text { Collection) }\end{array}$ & $2010-2018$ \\
\hline Rumination and depression & 29 & Psicodoc & $2010-2018$ \\
\hline Rumination and anxiety & 15 & Psicodoc & 2010-2018 \\
\hline Total & 11730 & & \\
\hline
\end{tabular}

\section{Selección de los estudios}

Una vez fueron eliminados los duplicados, se identificó el cumplimiento de los criterios de inclusión-exclusión de los documentos, en primer lugar, verificando el título de la investigación (cribado inicial), en segundo, la pertinencia según la lectura del abstract (selección para lectura completa).

\section{Proceso de extracción de datos y lista de datos}

Se extrajeron inicialmente, con el software Mendeley 8.1, los metadatos como título, resumen y referencia bibliográfica en formato APA. Después de pasar por el proceso de selección, se procedió a la lectura de los documentos en texto completo en PDF y se extrajeron de cada uno la cita, el país, el tamaño de la muestra (n), la edad de los participantes, el porcentaje de hombres y mujeres, el instrumento psicométrico usado, el trastorno internalizante evaluado, la correlación reportada y las principales conclusiones del estudio.

\section{Riesgo de sesgo}

Se contó con dos investigadores externos que puntuaron la aprobación (1), desaprobación (0), o pendiente de revisión (-), de cada uno de los artículos en texto completo en PDF elegibles, los 
acuerdos entre los investigadores con puntajes nominales sí/no permitieron asumir un control de sesgo de selección e inclusión de los estudios en la revisión sistemática. El procedimiento se llevó a cabo en una matriz de Excel en la que se añadieron columnas adicionales para cada evaluador.

\section{Resultados}

\section{Selección de estudios}

En la Figura 1 se presenta el proceso de identificación y selección de los artículos incluidos en la revisión sistemática. Se recolectaron un total de 11730 artículos de los cuales se eliminaron 3790 duplicados, teniendo como registros seleccionados 7892 artículos, se filtraron a partir de la revisión de los títulos y los resúmenes según los objetivos del estudio, con una exclusión final de 7741 documentos. Luego se evaluaron 103 artículos de texto completo para elegibilidad de los cuales se incluyeron 32 artículos, se tuvieron en cuenta artículos en idioma español e inglés.

\section{Figura 1}

Diagrama de flujo PRISMA

Registros identificados a través de la búsqueda en la base de datos $(\mathrm{n}=11730)$

Science Direct, Medline, PsyArticles and Proquest (Psychology database and Health, 6 Medical Collection)

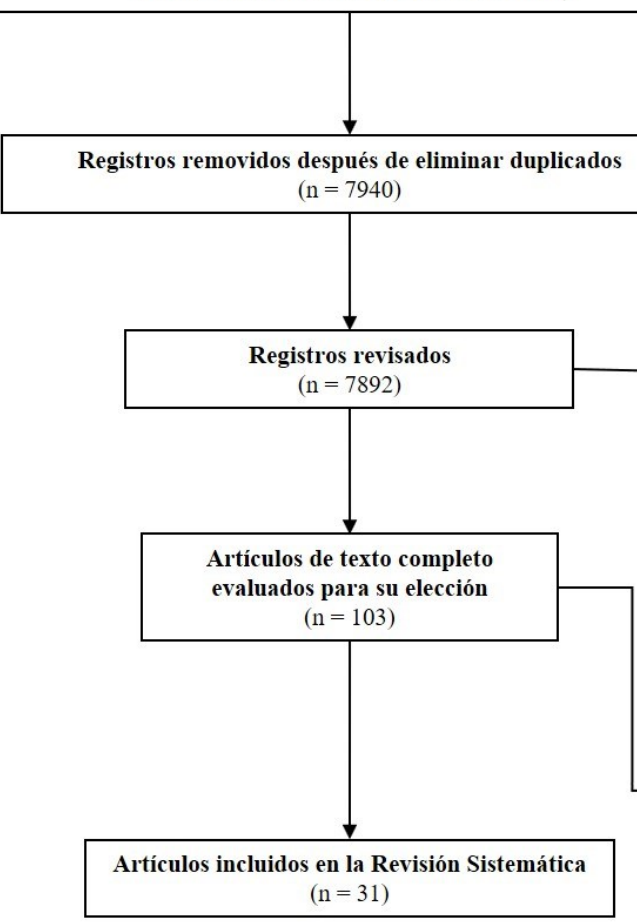

Registros adicionales identificados

en otras fuentes

$(n=44)$ Psicodoc

Registros excluidos $(n=48)$

$\begin{gathered}\text { Artículos de texto completo } \\ \text { excluidos } \\ (\mathrm{n}=72)\end{gathered}$
Razones: Consumo de SPA (1), biológico (6),
psicoterapia (7), únicamente un solo trastorno (19),
comprobación de teoría (1), perfeccionismo (7),
estudio de caso (1), procrastinación (1), revisión
teórica (1), no incluyó rumiación (1), procesos
cognitivos (3), pensamiento negativo (1), victimización
(1), ideación suicida (1), relaciones de pareja (1),
vulnerabilidad cognitiva (1), percepción física (1),
validación de test psicométrico (1), enfermedad física
(3), procesos sociales (1), apego (1), preocupación (3),
y estrategias de regulación emocional (1).




\section{Características del estudio}

De acuerdo con las temáticas analizadas y abordadas en la revisión sistemática, en cuanto a la rumiación desadaptativa y su influencia en la psicopatología internalizante, específicamente en depresión y ansiedad se encontraron nueve artículos (28.2\%), que informaron la influencia del aumento de la percepción negativa de ciertos estímulos que generan malestar y desencadenan pensamientos repetitivos. En tres artículos (9.4 $\%$ ) se identificó la influencia de la rumiación y el efecto negativo en psicopatología internalizante como depresión y ansiedad, mientras que siete artículos informaron sobre la influencia de la rumiación cognitiva únicamente en depresión
(21.8\%) y otros cinco artículos con los síntomas de ansiedad (15.6\%).

A su vez, se encontraron ocho artículos que aportaban al estudio al cumplir los criterios de inclusión, pero no indicaban estrictamente la psicopatología internalizante. De estos, tres artículos abordaron los estilos cognitivos (9.4 $\%)$, dos artículos informaron sobre la posible influencia positiva en la resolución de conflictos por medio de la rumiación adaptativa (6.2\%). Otros tres artículos sobre el afecto positivo y su influencia en la regulación de la rumiación (9.4 \%). En la Tabla 2, se resumen las características de los estudios incluidos en la revisión sistemática.

\section{Tabla 2}

Descripción de los estudios incluidos en la revisión sistemática

\begin{tabular}{|c|c|c|c|c|c|c|c|}
\hline Referencias & País & $\mathbf{n}$ & $\begin{array}{l}\text { Edad } \\
M(D E)\end{array}$ & $\begin{array}{l}\text { \% de } \\
\text { Mujeres y } \\
\text { Hombres }\end{array}$ & Tl evaluado & $\begin{array}{l}\text { Instrumentos Correlación } \\
\text { de medida }\end{array}$ & Conclusiones \\
\hline $\begin{array}{l}\text { Arditte et al. } \\
(2016) .\end{array}$ & $\begin{array}{l}\text { Estados } \\
\text { Unidos }\end{array}$ & $\begin{array}{l}\text { Estudio 1: } \\
126 \text { Estudio } \\
2: 228\end{array}$ & $\begin{array}{l}\text { Estudio 1: } \\
35.66 \text { (12.49) } \\
\text { Estudio 2: } \\
31.01(8.75)\end{array}$ & $\begin{array}{l}\text { Estudio } 1 . \\
M=61 \% \\
\text { Estudio } 2 . \\
M=50 \%\end{array}$ & $\begin{array}{l}\text { Espectro de } \\
\text { Ansiedad, } \\
\text { depresión y } \\
\text { TOC }\end{array}$ & $\begin{array}{l}\text { Estudio 1. } \\
\text { (RRS), (PEP- Ansiedad .57 } \\
\text { Q-R), (RPA), } \\
\text { (RTQ), (CES- } \\
\text {-D), } \\
\text { (DASS) } \\
\text { (SIAS). Estu- } \\
\text { dio 2. (RRS), } \\
\text { (PEPQ- } \\
\text { R), (RPA), } \\
\text { (DASS), } \\
\text { (DOCS) y } \\
\text { (BDD-SS). }\end{array}$ & $\begin{array}{l}\text { La rumiación se } \\
\text { considera un } \\
\text { desencadenante } \\
\text { clave de la } \\
\text { depresión y } \\
\text { ansiedad, y se } \\
\text { constituye como } \\
\text { mantenedor } \\
\text { de estas pro- } \\
\text { blemáticas. }\end{array}$ \\
\hline
\end{tabular}




\begin{tabular}{|c|c|c|c|c|c|c|c|c|}
\hline $\begin{array}{l}\text { Bartoskova } \\
\text { et al. (2018) }\end{array}$ & $\begin{array}{l}\text { Canadá, } \\
\text { Repúbli- } \\
\text { ca } \\
\text { Checa }\end{array}$ & 1414 & $19.60(3.40)$ & NR & $\begin{array}{l}\text { Depresión y } \\
\text { rumiación }\end{array}$ & $\begin{array}{l}\text { (RRS), (ARQ) } \\
\text { (BDI), (VAMP) } \\
\text { y (PHQ-9). }\end{array}$ & & $\begin{array}{l}\text { La rumiación } \\
\text { y la depresión } \\
\text { se relacionan } \\
\text { entre sí, ya que } \\
\text { la rumiación } \\
\text { funciona como } \\
\text { un procesador } \\
\text { de solución de } \\
\text { problemas, ex- } \\
\text { plica los motivos } \\
\text { porque los episo- } \\
\text { dios depresivos } \\
\text { se resuelven sin } \\
\text { tratamiento. }\end{array}$ \\
\hline $\begin{array}{l}\text { Black et al. } \\
(2013)\end{array}$ & $\begin{array}{l}\text { Estados } \\
\text { Unidos }\end{array}$ & $\begin{array}{l}302 / 100 / \\
92 / 86\end{array}$ & $13.58(0.56)$ & $\begin{array}{l}M=37.20 \% \\
H=62.79 \%\end{array}$ & $\begin{array}{l}\text { Rumiación y } \\
\text { depresión }\end{array}$ & $\begin{array}{l}\text { (DSQ), (RRS) } \\
\text { (RSQ) y } \\
\text { (SBB-DES). }\end{array}$ & NR & $\begin{array}{l}\text { Manifiesta que } \\
\text { la rumiación está } \\
\text { relacionada con } \\
\text { la aparición y el } \\
\text { mantenimiento } \\
\text { de los síntomas } \\
\text { depresivos en } \\
\text { adolescentes } \\
\text { que presentan } \\
\text { relaciones in- } \\
\text { terpersonales y } \\
\text { afectivas conflic- } \\
\text { tivas. }\end{array}$ \\
\hline $\begin{array}{l}\text { Brown et al. } \\
(2016)\end{array}$ & $\begin{array}{l}\text { Reino } \\
\text { Unido }\end{array}$ & 147 & 9.2 & $\begin{array}{l}H=46.93 \% \\
M=53.06 \%\end{array}$ & $\begin{array}{l}\text { Ansiedad y } \\
\text { depresión }\end{array}$ & $\begin{array}{l}\text { (CASI), } \\
\text { (CRSQ), } \\
\text { (SCAS) y } \\
\text { (SMFQ) }\end{array}$ & NR & $\begin{array}{l}\text { La presencia } \\
\text { de síntomas } \\
\text { de ansiedad y } \\
\text { depresión está } \\
\text { relacionada con } \\
\text { vulnerabilidades } \\
\text { cognitivas en los } \\
\text { niños basadas } \\
\text { en RC. }\end{array}$ \\
\hline
\end{tabular}




\begin{tabular}{|c|c|c|c|c|c|c|c|}
\hline $\begin{array}{l}\text { Chan et al. } \\
(2015)\end{array}$ & $\begin{array}{l}\text { Estados } 785 \\
\text { Unidos }\end{array}$ & $14.9(1.97)$ & $\begin{array}{l}H=44.96 \% \\
M=55.031 \%\end{array}$ & $\begin{array}{l}\text { Ansiedad, } \\
\text { depresión } \\
\text { rumiación, } \\
\text { catastrofi- } \\
\text { zación }\end{array}$ & $\begin{array}{l}\text { (SCARED), } \\
\text { (CERQ) y } \\
\text { (CNCEQ) }\end{array}$ & $\begin{array}{l}\text { Rumiación/ } \\
\text { Depresión } \\
\text { (.30) Catastr } \\
\text { fización/ } \\
\text { Depresión } \\
\text { (.47) Catastr } \\
\text { fización/ Ru- } \\
\text { miación (.45) }\end{array}$ & $\begin{array}{l}\text { Indica que los } \\
\text { niños presen- } \\
\text { - tan ansiedad y } \\
\text { catastrofización, } \\
\text { pero índices ba- } \\
\text { - jos de rumiación, } \\
\text { por el contrario, } \\
\text { las niñas que } \\
\text { presentan ansie- } \\
\text { dad y catastrofi- } \\
\text { zación sí presen- } \\
\text { tan índices altos } \\
\text { de rumiación. }\end{array}$ \\
\hline
\end{tabular}

Di Schiena et Estados Estudio 1. Estudio 1. al. (2013). Unidos 174 Estudio 2. 120

21.40 (3.44), Estudio 2. 22.45 (3.37)
M=78.17\% Rumiación y Estudio 1.
Rumiación/ Al recopilar los

Depresión

(.40) Ru-

miación/Ru-

miación (.45) estilos de ru-

\section{$\mathrm{H}=21.83 \%$} depresión

\section{The}

The Zung

Depression

Scale, (MiniCERTs),

(MDMQ) y

(IS). Estudio

2. (BDI).
Self-Rating

dos estudios, los miación (experimental-analítica/ experimentalconcreta), influyen en las estrategias de toma de decisiones, que llevan a la aparición o incremento de los síntomas depresivos.

$10.46(1.12) \quad M=100 \%$
Depresión, (CRSQ), ansiedad y rumiación (CRSQ),
(CASI), (BDI-
Y) y (BAI-Y)
Depresión/Ru- La rumiación miación (.63) está directaAnsiedad/Ru- mente relaciomiación (.57) nada con la Ansiedad/ depresión y no Depresión con la ansiedad. (.79)
Por el contrario, la sensibilidad a la ansiedad está relacionada únicamente con la ansiedad y no con la depresión. 
Fernández- España 312

Fernández et

al. (2014)
71.27 (6.99) $\mathrm{M}=71.7 \%$ $\mathrm{H}=28.3 \%$ Depresión y (CES-D), rumiación (RRS-Brief)

(LTS).
Gan et al. China 71 (2015)
$82.49(5.86)$

$\begin{array}{lll}\mathrm{M}=64,80 \% & \text { Depresión } & \text { UCLA-8 } \\ \mathrm{H}=35,20 \% & & \text { Loneliness } \\ & & \text { Scale, (RSS) } \\ & \text { y (CES-D). }\end{array}$

\author{
(.58) \\ Rumiación/ Los adultos \\ mayores que \\ presentan \\ tendencia a \\ la rumiación \\ y practican \\ actividades de \\ ocio, manifiestan \\ menores niveles \\ de síntomas \\ depresivos, a \\ comparación \\ de los adultos \\ mayores que no \\ realizan dichas \\ actividades \\ y utilizan la \\ rumiación como \\ una estrategia \\ de regulación \\ emocional \\ presentan \\ una mayor \\ vulnerabilidad \\ a los síntomas \\ depresivos.
}

Depresión
$(.30)$




\begin{tabular}{|c|c|c|c|c|c|c|c|}
\hline $\begin{array}{l}\text { Garrison et } \\
\text { al. (2014) }\end{array}$ & $\begin{array}{l}\text { Estados } 116 \\
\text { Unidos }\end{array}$ & $19.60(3.40)$ & $\begin{array}{l}M=62,93 \% \\
H=37,06 \%\end{array}$ & $\begin{array}{l}\text { Ansiedad d } \\
\text { apego }\end{array}$ & $\begin{array}{l}\text { (ECRS), } \\
\text { (DDI), (ERQ) } \\
\text { y (RRS). }\end{array}$ & $\begin{array}{l}\text { Ansiedad de } \\
\text { apego }(.23)\end{array}$ & $\begin{array}{l}\text { La evitación del } \\
\text { apego (miedo a } \\
\text { la dependencia) } \\
\text { se relacionó } \\
\text { negativamente } \\
\text { con las } \\
\text { tendencias de } \\
\text { divulgación y la } \\
\text { rumiación diaria } \\
\text { de eventos. La } \\
\text { ansiedad por } \\
\text { el apego (es } \\
\text { decir, el miedo } \\
\text { al rechazo) } \\
\text { se relacionó } \\
\text { positivamente } \\
\text { con ambas } \\
\text { medidas de } \\
\text { rumiación. }\end{array}$ \\
\hline $\begin{array}{l}\text { Geiger et al. } \\
(2010)\end{array}$ & $\begin{array}{l}\text { Estados } 396 \\
\text { Unidos }\end{array}$ & $19.9(2.7)$ & $\begin{array}{l}M=37.37 \% \\
H=62.12 \%\end{array}$ & Depresión & $\begin{array}{l}\text { (RRS), } \\
\text { (HS) y } \\
\text { (BDI). }\end{array}$ & $\begin{array}{l}\text { Depresión } \\
(.85)\end{array}$ & $\begin{array}{l}\text { Las personas } \\
\text { que presentan } \\
\text { altos niveles } \\
\text { de rumiación } \\
\text { incrementan su } \\
\text { sintomatología } \\
\text { depresiva, por } \\
\text { el contrario, las } \\
\text { personas que } \\
\text { presentan altos } \\
\text { niveles de espe- } \\
\text { ranza reportan } \\
\text { un alto estado } \\
\text { de ánimo. }\end{array}$ \\
\hline
\end{tabular}

Grant et al. Estados 127 (2010) Unidos

$M=76.37 \% \quad$ Ansiedad $\mathrm{H}=23.62 \%$
(CTAS), (RSQ), (TQPre, TQ-Post) y (PANAS).
NR

Los estudiantes de pregrado presentaron rumiación seguido a un evento donde se encontraban expuestos a la evaluación. Los participantes que presentaban pensamiento anticipatorio ante el examen manifestaron percibir mayor rumiación sobre el evento. 


\begin{tabular}{|c|c|c|c|c|c|c|c|c|}
\hline $\begin{array}{l}\text { Law et al. } \\
(2015)\end{array}$ & Canadá & 120 & $80.88(3.73)$ & $\begin{array}{l}M=77 \% \\
H=33 \%\end{array}$ & $\begin{array}{l}\text { Trastorno } \\
\text { de la } \\
\text { personali- } \\
\text { dad límite }\end{array}$ & $\begin{array}{l}\text { (PANAS), } \\
\text { (SCOPE), } \\
\text { (DES-IV), y } \\
\text { (SSFS). }\end{array}$ & NR & $\begin{array}{l}\text { Se verificaron } \\
\text { los efectos dife- } \\
\text { renciales de la } \\
\text { rumia inducida } \\
\text { por la ira y la } \\
\text { rumiación depre- } \\
\text { siva sobre el } \\
\text { afecto y las ex- } \\
\text { periencias auto- } \\
\text { conscientes. }\end{array}$ \\
\hline $\begin{array}{l}\text { Liu et al. } \\
\text { (2017) }\end{array}$ & China & 87 & $40.92(12.67)$ & $\begin{array}{l}M=64.36 \% \\
H=35.63 \%\end{array}$ & Depresión & $\begin{array}{l}\text { (RRS), } \\
\text { (HDRS) y } \\
\text { (AMT), }\end{array}$ & $\begin{array}{l}\text { Depresión } \\
(.54)\end{array}$ & $\begin{array}{l}\text { Se sugiere el } \\
\text { papel mediador } \\
\text { de la rumiación } \\
\text { en la relación } \\
\text { entre la memoria } \\
\text { autobiográfica y } \\
\text { la depresión, en } \\
\text { particular el sub- } \\
\text { tipo de rumiación } \\
\text { desadaptativa. }\end{array}$ \\
\hline $\begin{array}{l}\text { Liverant et a } \\
\text { (2011) }\end{array}$ & $\begin{array}{l}\text { Estados } \\
\text { Unidos }\end{array}$ & 60 & $34.6(13.02)$ & $\begin{array}{l}M=66.7 \% \\
H=33.3 \%\end{array}$ & $\begin{array}{l}\text { Depresión y } \\
\text { ansiedad }\end{array}$ & (BDI), (NEO) & $\begin{array}{l}\text { Depresión } \\
(.39) \\
\text { Ansiedad } \\
(.29)\end{array}$ & $\begin{array}{l}\text { Los hallazgos } \\
\text { sugieren que } \\
\text { la rumiación } \\
\text { es un posible } \\
\text { mecanismo de } \\
\text { acción para la } \\
\text { atención eficaz } \\
\text { y los tratamien- } \\
\text { tos basados en } \\
\text { la aceptación } \\
\text { y regulación } \\
\text { emocional. }\end{array}$ \\
\hline $\begin{array}{l}\text { Lo et al. } \\
\text { (2010) }\end{array}$ & $\begin{array}{l}\text { Hong } \\
\text { Kong }\end{array}$ & 72 & $19.47(1.37)$ & $\begin{array}{l}M=68.05 \% \\
H=31.94 \%\end{array}$ & Depresión & $\begin{array}{l}\text { (BDI-II), (VAS) } \\
\text { y (ASQ). }\end{array}$ & $\begin{array}{l}\text { Depresión } \\
(.66)\end{array}$ & $\begin{array}{l}\text { Se encontró una } \\
\text { relación positiva } \\
\text { entre el estilo } \\
\text { atributivo nega- } \\
\text { tivo y el nivel } \\
\text { de los síntomas } \\
\text { depresivos, en el } \\
\text { que el procesa- } \\
\text { miento en la ru- } \\
\text { mia interactuaba } \\
\text { con los síntomas } \\
\text { depresivos para } \\
\text { predecir el estilo } \\
\text { atributivo nega- } \\
\text { tivo. }\end{array}$ \\
\hline
\end{tabular}




\begin{tabular}{|c|c|c|c|c|c|c|c|c|}
\hline $\begin{array}{l}\text { McCracken } \\
\text { et al. (2014) }\end{array}$ & $\begin{array}{l}\text { Reino } \\
\text { Unido }\end{array}$ & 352 & $\begin{array}{l}47.3 \\
(11.69)\end{array}$ & $\begin{array}{l}M=66.9 \% \\
H=33.1 \%\end{array}$ & $\begin{array}{l}\text { Dolor cróni- } \\
\text { co, somati- } \\
\text { zación }\end{array}$ & $\begin{array}{l}\text { (EQ), (AAQ- } \\
\text { II), (CPAQ), } \\
\text { (PHQ-9), (SF- } \\
36)\end{array}$ & $\begin{array}{l}\text { Gravedad } \\
\text { dolor }(.25)\end{array}$ & $\begin{array}{l}\text { La rumiación } \\
\text { cognitiva se } \\
\text { asocia de ma- } \\
\text { nera integral } \\
\text { con diferentes } \\
\text { factores que } \\
\text { explican el do- } \\
\text { lor crónico y su } \\
\text { agravamien- } \\
\text { to: flexibilidad } \\
\text { psicológica, } \\
\text { aceptación, } \\
\text { funcionamiento } \\
\text { social y físico. }\end{array}$ \\
\hline
\end{tabular}

Macrynikola Estados 261

et al. (2017) Unidos

Malmberg et Holanda 417

al. (2015)

McEvoy et al. Aus(2013)
$20.3(4.4)$

$$
\mathrm{M}=81.6 \%
$$

$\mathrm{H}=18.4$

$\mathrm{M}=66.42 \%$

$\mathrm{H}=33.58 \%$
Depresión

(RRS) y (BDI) Fantasía tiem- Las fantasías pos 1 y $2=$ positivas su.30 y Valencia gieren que la de narrativas incubación es en tiempos 1 un mecanismo y $2=.12$

Depresión

(RRS) y (CES-D).

Rumiación/ Este estudio Depresión sugiere que los (.55) Insom- individuos con nio/Depresión presencia de (.44) y Ru- insomnio pueden miación/ In- presentar los somnio (.29) niveles más altos de rumia y niveles más altos de síntomas depresivos.

(RTQ-L,), Falta de Se sugiere que (MCQ-30), control y pen- las experiencias (TCQ), (CAQ), samientos previas (BAI) y (BDI- rumiativos .66 actúan como II). precipitante de la presencia de estados rumiativos y psicopatología internalizante. 


\begin{tabular}{|c|c|c|c|c|c|c|c|c|}
\hline $\begin{array}{l}\text { McLaughlin } \\
\text { et al. (2011) }\end{array}$ & $\begin{array}{l}\text { Estados } \\
\text { Unidos }\end{array}$ & $\begin{array}{l}\text { Adolescentes } \\
=1065 \text { Adul- } \\
\text { tos }=1317\end{array}$ & $\begin{array}{l}\text { Adolescentes = } \\
11-14 \text { Adultos= } \\
25-75\end{array}$ & $\begin{array}{l}M=48.8 \% \\
H=51.2 \%\end{array}$ & $\begin{array}{l}\text { Depresión y } \\
\text { ansiedad }\end{array}$ & $\begin{array}{l}\text { (CDI), } \\
\text { (MASC), } \\
\text { (CRSQ), } \\
\text { (BDI), (BAI) y } \\
\text { (RSQ). }\end{array}$ & $\begin{array}{l}\text { Depresión/ } \\
\text { Ansiedad } \\
(.67)\end{array}$ & $\begin{array}{l}\text { Se sugiere que } \\
\text { el tratamiento } \\
\text { de la rumia en } \\
\text { los enfoques } \\
\text { de tratamiento } \\
\text { transdiagnós- } \\
\text { tico aumentan la } \\
\text { probabilidad de } \\
\text { mejora para tras- } \\
\text { tornos emocio- } \\
\text { nales. }\end{array}$ \\
\hline $\begin{array}{l}\text { McLaughlin } \\
\text { et al. (2014) }\end{array}$ & $\begin{array}{l}\text { Estados } \\
\text { Unidos }\end{array}$ & 1.065 & $12.2(1.0)$ & $\begin{array}{l}M=48.8 \% \\
H=51.2 \%\end{array}$ & $\begin{array}{l}\text { Depresión y } \\
\text { ansiedad }\end{array}$ & $\begin{array}{l}\text { (CRSQ), } \\
\text { (RPEQ), (CDI) } \\
\text { y (MASC). }\end{array}$ & $\begin{array}{l}\text { Depresión/ } \\
\text { Rumiación } \\
(.19) \\
\text { Ansiedad/Ru- } \\
\text { miación (.15) }\end{array}$ & $\begin{array}{l}\text { La rumia predice } \\
\text { aumentos so- } \\
\text { bre tiempo en } \\
\text { el comporta- } \\
\text { miento agresivo, } \\
\text { y la agresión } \\
\text { se asoció con } \\
\text { aumentos en } \\
\text { la rumia a nivel } \\
\text { temporal. La } \\
\text { rumiación medió } \\
\text { completamente } \\
\text { la asociación } \\
\text { longitudinal de la } \\
\text { agresión con la } \\
\text { ansiedad subsi- } \\
\text { guiente. }\end{array}$ \\
\hline $\begin{array}{l}\text { Mezulis et al. } \\
(2011)\end{array}$ & $\begin{array}{l}\text { Estados } \\
\text { Unidos }\end{array}$ & 301 & $\begin{array}{l}H=13.52(0.33) \\
M=15.50(0.33)\end{array}$ & $\begin{array}{l}M=52.49 \% \\
H=47.51 \%\end{array}$ & Depresión & $\begin{array}{l}\text { (IBQ), } \\
\text { (RRS), (RSQ) } \\
\text { y (CDI). }\end{array}$ & $\begin{array}{l}\text { Rumiación } \\
\text { (.17) } \\
\text { Depresión } \\
(.10)\end{array}$ & $\begin{array}{l}\text { Se reportaron } \\
\text { factores de } \\
\text { vulnerabilidad } \\
\text { afectiva y cogni- } \\
\text { tiva en la predic- } \\
\text { ción síntomas } \\
\text { depresivos y la } \\
\text { diferencia de } \\
\text { géneros en la } \\
\text { depresión en la } \\
\text { adolescencia. }\end{array}$ \\
\hline
\end{tabular}




\begin{tabular}{|c|c|c|c|c|c|c|c|}
\hline $\begin{array}{l}\text { Michl et al. } \\
(2013)\end{array}$ & $\begin{array}{l}\text { Estados } \\
\text { Unidos }\end{array}$ & $\begin{array}{l}\begin{array}{l}\text { Adoles- } \\
\text { centes=1065 }\end{array} \\
\text { Adultos=1132 }\end{array}$ & $\begin{array}{l}M=48.8 \% \\
H=51.2 \%\end{array}$ & $\begin{array}{l}\text { Depresión y } \\
\text { ansiedad }\end{array}$ & $\begin{array}{l}\text { (CRSQ), } \\
\text { (CDI) y } \\
\text { (MASC). }\end{array}$ & $\begin{array}{l}\text { Ansiedad/ } \\
\text { Rumiación } \\
(.63)\end{array}$ & $\begin{array}{l}\text { Se encontró que } \\
\text { la exposición a } \\
\text { eventos vitales } \\
\text { estresores se } \\
\text { asoció longitu- } \\
\text { dinalmente con } \\
\text { el incremento } \\
\text { en la rumiación. } \\
\text { Además, se re- } \\
\text { portó que media } \\
\text { la aparición de } \\
\text { síntomas ansio- } \\
\text { sos en ambas } \\
\text { muestras, y me- } \\
\text { dia la depresión } \\
\text { en los adultos. }\end{array}$ \\
\hline
\end{tabular}

Nikcevic et Estados 273

al. (2014) Unidos

Raes (2010) Bélgica 271
22.2(5.7)

$$
M=20 \%
$$

$\mathrm{H}=79.1 \%$
Depresión

(PSS), (RRS- Rumiación 10), (PSWQ), (0.61) y (HADS).
La rumiación y la preocupación se asoció positiva y significativamente con los síntomas depresivos, además reportaron que las situaciones percibidas como amenazantes aumentan significativamente la presencia de la rumiación.

Los resultados indicaron que solamente la rumiación fue un mediador significativo en la relación entre autocompasión y la depresión. (RRS), y
(PSWQ). II), (STAI-T)

$\mathrm{M}=78.96 \%$ Depresión y (SCS), (BDI- NR $\mathrm{H}=21.04 \%$ ansiedad

(PSWQ) 


\begin{tabular}{|c|c|c|c|c|c|c|c|}
\hline $\begin{array}{l}\text { Rood et al. } \\
(2012)\end{array}$ & $\begin{array}{l}\text { Estados } 160 \\
\text { Unidos }\end{array}$ & $14.45(1.10)$ & $\begin{array}{l}\mathrm{H}=49.4 \% \\
\mathrm{M}=50.1 \%\end{array}$ & Estrés & $\begin{array}{l}\text { (SRRS-C), } \\
\text { (CDI), (VAS) }\end{array}$ & NR & $\begin{array}{l}\text { Reportaron que } \\
\text { la reevaluación } \\
\text { positiva (pensar } \\
\text { en los beneficios } \\
\text { y crecimiento } \\
\text { personal) causó } \\
\text { un incremento } \\
\text { en el afecto } \\
\text { positivo y dismi- } \\
\text { nución del afecto } \\
\text { negativo en } \\
\text { comparación con } \\
\text { la rumiación, el } \\
\text { distanciamiento } \\
\text { y la no acep- } \\
\text { tación. }\end{array}$ \\
\hline
\end{tabular}

Ruscio et al. Estados Control=33 Control=28.61 Control: Estrés, MDD, GAD, NR (2015)
Unidos Ansiedad generalizada $=36$

Depresión mayor $=38$ Comórbidos $=38$
$(10.42) \mathrm{GAD}=$ $31.62(9.24)$ $\mathrm{MDD}=36.38$

(12.33)

Comórbido= 33.60(11.35)
$M=66.7 \%$

$\mathrm{H}=54.5 \%$

GAD:

$\mathrm{M}=83.3 \%$

$\mathrm{H}=63.9 \%$

MDD:

$M=71.1 \%$

$\mathrm{H}=54.1$

Comórbido:

$M=52.6$

$\mathrm{H}=56.8$ $\begin{array}{ll}\text { Estrés, } & \text { MDD, GAD, } \\ \text { depresión } & \text { MDD, GAD. }\end{array}$

mayor y

trastorno de

ansiedad

generali-

zada.
La rumiación que se correlacionó con el estrés fue más perjudicial para los diagnosticados que los individuos controles. Reportaron que entre más intensos los síntomas clínicos más graves $y$ más persistentes fueron los indicadores de vulnerabilidad temperamental por trastornos emocionales. 


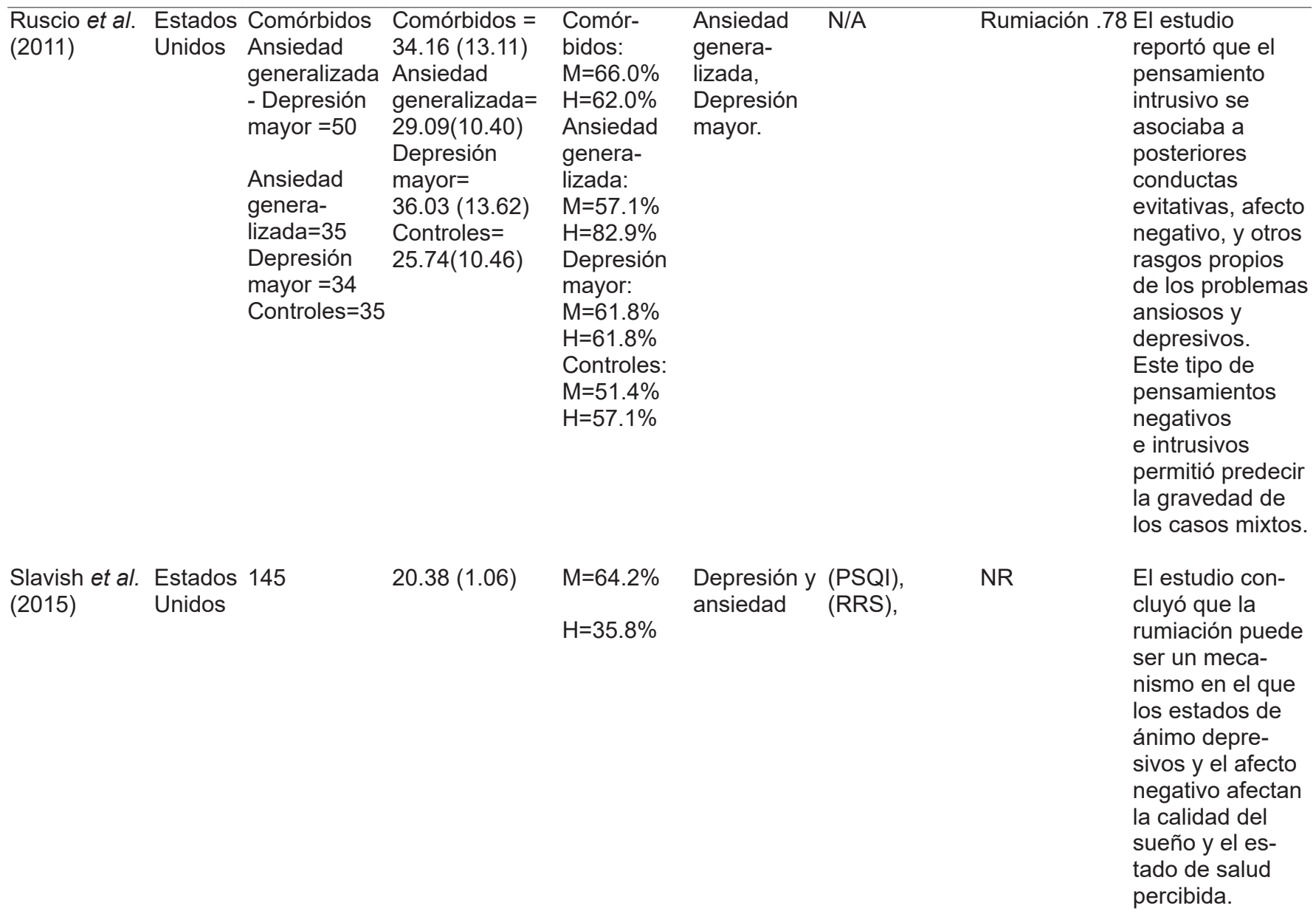


Evaluación de la calidad metodológica y riesgo de sesgos

Se realizó un control de sesgos para la selección de los artículos, en la que participaron dos investigadores externos, que verificaron el aporte identificado, los instrumentos usados, si se presentaban correlaciones entre las variables cognitivas y psicopatológicas internalizantes, y una conclusión. De los 103 artículos analizados, se evaluó un acuerdo interjueces a partir de las puntuaciones sí/no de dos jurados. Se obtuvo para exclusión un $63 \%$ y un $37 \%$ seleccionados para la extracción de aportes.

\section{Discusión}

En los estudios revisados, se encontraron diversas posturas con respecto a la rumiación cognitiva y su asociación con la psicopatología internalizante. Los hallazgos se analizaron desde el estilo cognitivo, la rumiación adaptativa y desadaptativa, su relación con el afecto positivo y negativo, y con respecto a las psicopatologías en el espectro ansioso, depresivo, somático y mixto.

\section{Estilo cognitivo}

Se identificó que los pensamientos rumiativos influyen en la aparición de los síntomas depresivos como la falta de sueño, anhedonia y labilidad emocional, y que los estilos cognitivos de los participantes podrían ser parte de los predisponentes para la aparición de psicopatología internalizante (Gan et al., 2015). Estos estilos cognitivos darían cuenta del papel de los eventos desencadenantes y la percepción individual de cada sujeto, frente a los diversos estímulos que pueden elicitar los pensamientos rumiativos (Chan et al., 2015).

Por ejemplo, se identificó que existen diferencias individuales en la trayectoria de la rumiación seguida de un evento percibido como negativo. En una muestra de 127 estudiantes universitarios se encontró que la rumiación sobre las posibles consecuencias que se podrían presentar frente a la evaluación, según los estilos cognitivos como el impulsivo y el reflexivo, aumentaron o disminuyeron la trayectoria del efecto negativo ante la rumiación (Grant y Beck, 2010). En síntesis, se obtuvo evidencia acerca de las relaciones entre rumiación cognitiva, los trastornos de ansiedad, depresión y somatización, especialmente en personas con ansiedad y depresión explicados a partir del estilo cognitivo.

\section{Rumiación adaptativa}

La rumiación reflexiva se asocia a un constructo funcional, ya que le permite al individuo solucionar problemas y aliviar estados de ánimo bajos, mientras que la rumiación negativista es una estrategia disfuncional acompañada de afecto negativo, considerando este como un factor de vulnerabilidad para la depresión y los trastornos de ansiedad. Al evaluar los componentes de la solución de problemas, se encontró que la rumiación adaptativa permite comprender la evolución de los episodios depresivos sin necesidad de un tratamiento médico, aumentando la regulación 
emocional por medio del análisis causal del pensamiento (Bartoskova et al., 2018).

\section{Rumiación y afecto positivo}

Se examinó la relación entre la rumiación y las estrategias de la regulación emocional en pacientes diagnosticados con depresión unipolar; los investigadores reportaron que la rumiación se asoció positivamente con otros tipos de supresión de malestar. Los resultados del estudio indicaron que la rumiación podría ser considerada como estrategia adaptativa para suprimir experiencias de emoción negativa, con un posible mecanismo de acción basado en la aceptación en el tratamiento de la depresión (Liverant et al., 2011). Asimismo, en un estudio de comparación de los efectos de la rumiación, la reevaluación positiva, el distanciamiento y la aceptación de los estados afectivos en adolescentes, la reevaluación positiva causó un aumento significativo en el efecto positivo y una disminución en el efecto negativo con respecto a la rumia, estos hallazgos indican que la reevaluación positiva hace parte de una estrategia de afrontamiento adaptativa a corto plazo (Rood et al., 2012).

Adicionalmente, en un análisis de los efectos de la rumiación y la esperanza, un estudio planteó la hipótesis que la esperanza podría moderar la relación entre la rumia y los síntomas depresivos. Se reportaron efectos en la rumia y la esperanza con asociaciones bidireccionales, en los que efectos de la esperanza podrían ser un factor protector para reducir los niveles de rumiación y sintomatología depresiva (Geiger y Kwon, 2010). En síntesis, en los estudios documentados se evidenció la relación entre rumiación cognitiva positiva frente a los incrementos en habilidades de resolución de problemas, la flexibilidad cognitiva y los estilos de afrontamiento funcionales.

\section{Rumiación desadaptativa}

La rumiación desadaptativa se ha considerado como uno de los predictores con mayor evidencia reciente de la psicopatología internalizante, los estudios encontrados indican que puede estar presente a lo largo del ciclo vital y parece presentarse con mayor frecuencia en mujeres. En un estudio con niños depresivos, las asociaciones específicas y compartidas entre las subescalas de sensibilidad a la ansiedad y rumiación se asociaron también a los síntomas depresivos, lo que indicó vulnerabilidades cognitivas que estarían mediadas por la rumiación (Brown et al., 2016). En los adolescentes que desarrollan depresión, se encontró evidencia en la que tienden a rumiar sobre sus experiencias interpersonales negativas, lo cual aumentó la sintomatología depresiva y la conducta adictiva (Black y Pössel, 2013).

En otra investigación se examinó la relación entre la rumiación, la distorsión cognitiva, la ansiedad y la depresión entre estudiantes universitarios, en los que se encontraron diferencias significativas entre hombres y mujeres, ya que las mujeres tienden a presentar mayor rumiación y mayor presencia 
de psicopatología internalizante (Basha, 2015). Igualmente, en personas de la tercera edad, en un estudio se determinó que la rumiación juega un papel moderador para la sintomatología depresiva, siendo este un perfil de riesgo en personas de la tercera edad, en donde la regulación emocional puede percibirse como disfuncional frente a los estados rumiativos (Fernández-Fernández et al., 2014).

De esta manera, se destaca que la rumiación puede ser el precipitante para generar un trastorno afectivo dado su papel mediador en los síntomas ansiosos y depresivos. En esta medida, su papel mantenedor de las psicopatologías internalizantes se constituye como evidencia de la vulnerabilidad y mantenimiento de síntomas depresivos de acuerdo a eventos negativos, y los estados rumiativos específicos (Grassia y Gibb, 2008).

En síntesis, la rumiación cognitiva desadaptativa actuaría como un predictor clave de la psicopatología internalizante; conlleva malestar y disfuncionalidad en las diversas áreas de ajuste, teniendo como predisponente vulnerabilidades psicológicas y ambientales; va acompañada de dificultades en la resolución de problemas adecuados, y resulta inflexible en los patrones de pensamiento. Además, parece presentarse diferencialmente entre hombres y mujeres, mientras que por edades no resultan claras las diferencias dadas las conclusiones revisadas.

\section{Rumiación y afecto negativo}

Se ha sugerido que la aparición de RC incrementa la respuesta de afecto negativo en las personas vulnerables a las psicopatologías internalizantes. McEvoy et al. (2013) en un estudio correlacional encontraron que existen mecanismos comunes que impulsan la rumiación desadaptativa antes y después de un factor estresante, que conlleva a un aumento de los niveles del afecto negativo. En otro estudio, al revisar los efectos de la rumiación sobre los factores relacionados con experiencias conscientes en ira y depresión, incluyendo el afecto negativo y las características del trastorno límite de la personalidad, se evidenció que tanto en la ira como en la depresión se presenta un aumento significativo del efecto negativo $\mathrm{y}$ la disminución del positivo (Law y Chapman, 2015). Al respecto, se ha considerado a la supresión del afecto negativo y la aceptación de la vivencia de las emociones como dos estrategias claves para la regulación emocional; sin embargo, los hallazgos acerca de la asociación entre la rumiación y otros tipos de supresión, favorecen la aparición de la psicopatología dada la interferencia entre aceptación y control de los síntomas (Liverant et al., 2011).

\section{Psicopatología internalizante y rumiación cognitiva}

\section{Depresión}

La rumiación se asocia directamente a la depresión, como una variable de elevada vulnerabilidad cognitiva que la desarrolla y mantiene, dado que ante la presencia de un 
evento negativo se incrementa la rumiación cognitiva y como consecuencia proviene la sintomatología depresiva, a diferencia de otras variables transdiagnósticas internalizantes como la sensibilidad a la ansiedad (Epkins et al., 2013). Se ha documentado que la rumiación cognitiva media la aparición de estados emocionales negativos como la ira y la culpa. Por ejemplo, ante emociones como la culpa, aparecen recuerdos de un evento y posteriormente la dificultad en controlar el pensamiento, lo que constituye evidencia del factor rumiación en la aparición y mantenimiento de la sintomatología depresiva e ira (Law y Chapman, 2015).

En un estudio longitudinal realizado desde el nacimiento hasta la adolescencia, se buscó examinar las posibles asociaciones entre afectividad negativa, rumiación y síntomas depresivos en jóvenes, según informes de las madres de los participantes. Los análisis de los datos indicaron que la rumiación medió significativamente la asociación afecto negativo y depresión a lo largo del seguimiento del estudio, con una diferencia significativa para las niñas. Concluyeron los investigadores que se trata de un factor de vulnerabilidad cognitiva y afectiva que explicaría la depresión en la infancia y adolescencia (Mezulis et al., 2011).

En otro estudio con jóvenes, se encontró que el estrés percibido, la rumia y la preocupación se asociaron positiva y significativamente con los síntomas depresivos y que el estrés percibido se asoció positiva y significativamente con la rumia y la preocupación. Un análisis de moderación confirmó que el pensamiento recurrente negativo moderó la relación entre el estrés percibido y los síntomas depresivos (Nikcevic et al., 2014).

A nivel explicativo, se ha sugerido que el estilo cognitivo negativo cumple un papel moderador en la aparición de sintomatología depresiva, esto depende del tipo de procesamiento de la información percibida del medio que surgirá como un modelo predictor al estilo cognitivo negativo (Lo et al., 2010). Además, se ha identificado que un factor desencadenante de la rumiación es la memoria autobiográfica, debido a que todo evento pasado que se percibe como negativo y es traído al momento presente puede generar la presencia de rumiación y como consecuencia la sintomatología depresiva (Liu et al., 2017).

Otra alternativa se ha asociado a las fantasías de los seres humanos, estos crean una serie de pensamiento hacia el futuro que se asemejan a un estilo de respuesta cognitiva desadaptada, y se intensifica la vulnerabilidad para la aparición de sintomatología depresiva, al ser un pensamiento repetitivo dirigido hacia las expectativas futuras. Ante fantasías negativas, las personas vulnerables no perciben un contraste entre su fantasía y la realidad de ocurrencia de la misma (Macrynikola et al., 2017).

Estas alteraciones también han sido asociadas al insomnio. En un estudio transversal con 417 participantes se concluyó que el insomnio media la relación entre la rumiación y los síntomas depresivos (Malmberg y 
Larsen, 2015). Al indagar si la RC explica las asociaciones entre el estado de ánimo depresivo y la calidad del sueño y la salud autoinformada, se obtuvo evidencia que la RC puede ser un mecanismo psicológico por el cual el estado de ánimo negativo conduce a un sueño deficiente y una salud percibida empeorada (Slavish y Graham-Engeland, 2015).

\section{Ansiedad}

La relación entre $\mathrm{RC}$ y ansiedad obtuvo una menor evidencia en los estudios incluidos en la revisión sistemática. Se encontró que la ansiedad por apego se relacionaba positivamente con la rumiación de los eventos emocionales diarios, además, fue un factor predictivo positivo, aunque, la mediación no fue lo suficientemente fuerte como para ser estadísticamente significativa a nivel bivariado (Garrison et al., 2014).

Se encontró que los estudios revisados indicaron que la $\mathrm{RC}$ se concibe como un proceso transdiagnóstico asociado a la depresión, ansiedad, abuso de sustancias, ingesta excesiva y comportamientos autoagresivos. Sin embargo, la evidencia se centra en los ansiosos y depresivos combinados. Al respecto, en un estudio se examinaron las experiencias ambientales sociales y su relación con la rumiación, se encontró que la exposición autoinformada de eventos estresantes vitales se asocian longitudinalmente con una mayor rumiación. Además, se presentó una mediación longitudinal entre los factores de estrés y los síntomas de ansiedad, y una relación entre los eventos cotidianos y los síntomas depresivos en adultos (Michl et al., 2013).

En cuanto a los efectos mediadores de la rumiación y la autocompasión en la depresión y la ansiedad, se encontró una relación con depresión, en la que únicamente la rumiación surgió como un mediador significativo. En ansiedad, la inquietud y la preocupación surgieron como mediadores significativos, pero el afecto fue un mediador estadísticamente mayor que la inquietud en la preocupación. Al parecer, la autocompasión tendría efectos amortiguadores en la depresión y la ansiedad a través de sus efectos en el pensamiento repetitivo (Raes, 2010).

\section{Somatización}

Se encontró únicamente un estudio en el que se reportó una asociación que diera cuenta de este indicador internalizante. En este estudió se concluyó que la rumiación cognitiva se asociaba de manera integral con diferentes factores que explicaban el dolor crónico y su agravamiento: entre ellos la flexibilidad psicológica y la aceptación, y por otra parte el funcionamiento social y el desempeño físico, como parte de un modelo clínico de defusión cognitiva (McCracken et al., 2014).

\section{Limitaciones}

Esta revisión sistemática presenta algunas limitaciones que hubieran sido de aporte al proceso de investigación. En un primer lugar, no se tuvieron en cuenta los artículos con variables de tipo biológico que estaban relacionados con 
patologías internalizantes y su relación con las estructuras cerebrales e implicaciones en el sistema nervioso; esta información habría podido complementar el análisis sistemático de la rumiación frente a las patologías internalizantes, y la conexión directa e indirecta con las estructuras que se ven comprometidas en el funcionamiento cerebral. Para futuras revisiones sistemáticas, se puede contemplar incluir la variable biológica.

Un segundo aspecto, fue no haber incluido en la revisión sistemática investigaciones relacionadas con causalidad a partir del tratamiento, ya que en la revisión no se contempló la efectividad de procedimientos (aplicación de tratamiento) sino la asociación entre rumiación, ansiedad y depresión. Estas investigaciones pueden proporcionar aporte científico donde se especifique si la manera en como se interviene la problemática permite inferir un papel causal según las modificaciones en la rumiación y cómo repercute a la mejora de una psicopatología internalizante. Se sugiere realizar una revisión sistemática posterior que relacione la rumiación con la efectividad de los tratamientos de patologías internalizantes, incluyendo un metanálisis, en el que se identifiquen posibles moderadores como la edad, el sexo, el país en el que se realizó el estudio, y otros factores culturales que puedan explicar las variaciones en los reportes consultados.

\section{Conclusión}

Se realizó una revisión sistemática de la literatura en la que se encontraron evidencias de estudios sobre la relación entre la rumiación cognitiva y la psicopatología internalizante. La información hallada proporciona datos relevantes, por un lado, sobre la importancia de concebir la rumiación como un factor mediador para la aplicación de tratamientos transdiagnósticos de los trastornos emocionales; por otro lado, en su mayoría los estudios profundizan en que la rumiación funciona como variable desencadenante o mantenedora de la aparición de psicopatología internalizante (depresión y ansiedad). Esta variable transdiagnóstica requiere ser contemplada como un mediador psicológico clave para realizar propuestas de intervención para el tratamiento de las psicopatologías internalizantes.

\section{Referencias $^{1}$}

Achenbach, T. M., Ivanova, M. Y., Rescorla, L. A., Turner, L. V. y Althoff, R. R. (2016). Internalizing/Externalizing Problems: Review and Recommendations for Clinical and Research Applications. Journal of the American Academy of Child \& Adolescent Psychiatry, 55(8), 647-656. https://doi. org/10.1016/j.jaac.2016.05.012

Alcázar-Olán, J., Merckel-Niehus, N., ToscanoBarranco, D., Barrera-Muñoz, O. y Proal-Sánchez, M. F. (2018). Adherencia

1 *Estudios incluidos en la revisión sistemática. 
al tratamiento $\mathrm{y}$ efectividad de un programa cognitivo conductual sobre la rumiación y expresión de la ira. Revista de Psicopatología y Psicología Clínica, 23(1), 13-23. https://doi.org/10.5944/ rppc.vol.23.num.1.2018.19248

*Arditte, K. A., Shaw, A. M. y Timpano, K. R. (2016). Repetitive negative thinking: a transdiagnostic correlate of affective disorders. Journal of Social and Clinical Psychology, 35(3), 181-201. https://doi. org/10.1521/jscp.2016.35.3.181

*Basha, S. E. (2015). Rumination, cognitive distortion, and its relation to anxiety and depression symptoms. Indian Journal of Health and Wellbeing, 6(11), 1049-1061. http://www.i-scholar.in/index.php/ijhw/ article/view/147297

*Bartoskova, M., Sevcikova, M., Durisko, Z., Maslej, M. M., Barbic, S. P., Preiss, M. y Andrews, P. W. (2018). The form and function of depressive rumination. Evolution and Human Behavior, 39(3), 277289. https://doi.org/10.1016/j.evolhumbehav.2018.01.005

Belloch, A. (2012). Propuestas para un enfoque transdiagnóstico de los trastornos mentales y del comportamiento: evidencia, utilidad y limitaciones. Revista de Psicopatología y Psicología Clínica, 17(3), 295-311. https://www.aepcp.net/arc/08_2012_n3_ belloch.pdf

*Black, S. W. y Pössel, P. (2013). The combined effects of self-referent information processing and ruminative responses on adolescent depression. Journal of Youth and Adolescence, 42(8), 1145-1154. https://doi.org/10.1007/s 10964-0129827-y

*Brown, H. M., Meiser-Stedman, R., Woods, H. y Lester, K. J. (2016). Cognitive vulnerabilities for depression and anxiety in childhood: Specificity of anxiety sensitivity and rumination. Behavioural and Cognitive Psychotherapy, 44(1), 30-42. https:// doi.org/10.1017/S1352465814000472

*Chan, S. M., Chan, S. K. y Kwok, W. W. (2015). Ruminative and catastrophizing cognitive styles mediate the association between daily hassles and high anxiety in Hong Kong adolescents. Child Psychiatry And Human Development, 46(1), 5766. https://doi.org/10.1007/s10578-0140451-9

DeJong, H., Fox, E. y Stein, A. (2016). Rumination and postnatal depression: A systematic review and a cognitive model. Beha-viour Research and Therapy, 82, 38-49. https://doi.org/10.1016/j. brat.2016.05.003

*Di Schiena, R., Luminet, O., Chang, B. y Philippot, P. (2013). Why are depressive individuals indecisive? different modes of rumination account for indecision in nonclinical depression. Cognitive Therapy and Research, 37(4), 713-724. https://doi. org/10.1007/s10608-012-9517-9 
*Epkins, C. C., Gardner, C. y Scanlon, N. (2013). Rumination and anxiety sensitivity in preadolescent girls: independent, combined, and specific associations with depressive and anxiety symptoms. Journal of Psychopathology and Behavioral Assessment, 35(4), 540-551. https://doi. org/10.1007/s10862-013-9360-7

*Fernández-Fernández, V., Márquez-González, M., Losada-Baltar, A. y Romero-Moreno, R. (2014). Frequency of leisure activities and depressive symptomatology in elderly people: the moderating role of rumination. International Psychogeriatrics, 26(2), 297-305. https://doi.org/10.1017/ S1041610213001877

*Gan, P., Xie, Y., Duan, W., Deng, Q. y Yu, X. (2015). Rumination and loneliness independently predict six-month later depression symptoms among Chinese elderly in nursing homes. PLoS One, 10(9). https:// doi.org/10.1371/journal.pone.0137176

*Garrison, A. M., Kahn, J. H., Miller, S. A. y Sauer, E. M. (2014). Emotional avoidance and rumination as mediators of the relation between adult attachment and emotional disclosure. Personality and Individual Differences, 70, 239-245. https://doi. org/10.1016/j.paid.2014.07.006

Gaviria, A., Correa, L. F., Dávila, C. E., Burgos, G., Osorio, E. D. y Valderrama, J. (2017). Guía metodológica para el observatorio nacional de salud mental. Ministerio de salud y protección social, 1, 3-47. http://
onsaludmental.minsalud.gov.co/Paginas/ Inicio.aspx

*Geiger, K. A. y Kwon, P. (2010). Rumination and depressive symptoms: Evidence for the moderating role of hope. Personality and Individual Differences, 49(5), 391-395. https://doi.org/10.1016/j. paid.2010.04.004

González, M., Ibáñez, I. y Barrera, A. (2017). Rumiación, preocupación y orientación negativa al problema: procesos transdiagnósticos de los trastornos de ansiedad, de la conducta alimentaria y del estado de ánimo. Acta Colombiana de Psicología, 20(2), 42-52. https://doi. org/10.14718/ACP.2017.20.2.3

*Grant, D. M. y Beck, J. G. (2010). What predicts the trajectory of rumination? A prospective evaluation. Journal of Anxiety Disorders, 24(5), 480-486. https://doi. org/10.1016/j.janxdis.2010.03.004

Grassia, M. y Gibb, B. E. (2008). Rumination and prospective changes in depressive symptoms. Journal of Social and Clinical Psychology, 27(9), 931-948. https://doi. org/10.1521/jscp.2008.27.9.931

*Law, K. C. y Chapman, A. L. (2015). Borderline personality features as a potential moderator of the effect of anger and depressive rumination on shame, self-blame, and self-forgiveness. Journal of Behavior Therapy and Experimental Psychiatry, 46, 27-34. https://doi. org/10.1016/j.jbtep.2014.07.008 
*Liu, Y., Yu, X., Yang, B., Zhang, F., Zou, W., Na, A., Zhao, X. y Yin, G. (2017). Rumination mediates the relationship between overgeneral autobiographical memory and depression in patients with major depressive disorder. BMC Psychiatry, 17, 1-7. https://doi.org/10.1186/s12888-017$1264-8$

*Liverant, G. I., Kamholz, B. W., Sloan, D. M. y Brown, T. A. (2011). Rumination in Clinical Depression: A Type of Emotional Suppression? Cognitive Therapy and Research, 35(3), 253-265. https://doi. org/10.1007/s10608-010-9304-4

*Lo, C. S. L., Ho, S. M. Y. y Hollon, S. D. (2010). The Effects of Rumination and Depressive Symptoms on the Prediction of Negative Attributional Style Among College Students. Cognitive Therapy and Research, 34(2), 116-123. https://doi. org/10.1007/s10608-009-9233-2

*Macrynikola, N., Goklani, S., Slotnick, J. y Miranda, R. (2017). Positive futureoriented fantasies and depressive symptoms: Indirect relationship through brooding. Consciousness and Cognition, 51, 1-9. https://doi.org/10.1016/j. concog.2017.02.013r

*Malmberg, M.y Larsen, J.K. (2015). Depressive Symptoms: The Interaction between Rumination and Self-Reported Insomnia. Depression Research and Treatment 10, 1-4 https://doi.org/10.1155/2015/150828

*McCracken, L. M., Barker, E. y Chilcot, J.
(2014). Decentering, rumination, cognitive defusion, and psychological flexibility in people with chronic pain. Journal of Behavioral Medicine, 37(6), 12151225. https://doi.org/10.1007/s10865014-9570-9

*McEvoy, P. M., Moulds, M. L. y Mahoney, A. E. J. (2013). Mechanisms driving pre- and post-stressor repetitive negative thinking: Metacognitions, cognitive avoidance, and thought control. Journal of Behavior Therapy and Experimental Psychiatry, 44(1), 84-93. https://doi.org/10.1016/j. jbtep.2012.07.011

*McLaughlin, K. A. y Nolen-Hoeksema, S. (2011). Rumination as a transdiagnostic factor in depression and anxiety. Behaviour Research and Therapy, 49(3), 186-193. https://doi.org/10.1016/j.brat.2010.12.006

*McLaughlin, K. A., Aldao, A., Wisco, B. E. y Hilt, L. M. (2014). Rumination as a transdiagnostic factor underlying transitions between internalizing symptoms and aggressive behavior in early adolescents. Journal of Abnormal Psychology, 123(1), 13-23. https://doi. org/10.1037/a0035358

*Mezulis, A. H., Priess, H. A. y Hyde, J. S. (2011). Rumination mediates the relationship between infant temperament and adolescent depressive symptoms. Depression Research and Treatment, 2011, 487873. https://doi. org/10.1155/2011/487873 
*Michl, L. C., McLaughlin, K. A., Shepherd, K. y Nolen-Hoeksema, S. (2013). Rumination as a mechanism linking stressful life events to symptoms of depression and anxiety: Longitudinal evidence in early adolescents and adults. Journal of Abnormal Psychology, 122(2), 339-352. https://doi.org/10.1037/a0031994

Moher, D., Liberati, A., Tetzlaff, J., Altman, D. G. y The PRISMA Group (2009). Preferred Reporting Items for Systematic Reviews and Meta-Analyses: The PRISMA Statement. PLoS Medicine, 6(7), e1000097. https://doi.org/10.1371/ journal.pmed 1000097

Morrison, R. y O’Connor, R. C. (2008). A Systematic Review of the Relationship Between Rumination and Suicidality. Suicide and Life-Threatening Behavior, 38(5), 523-538. https://doi.org/10.1521/ suli.2008.38.5.523

*Nikcevic, A. V, Caselli, G., Green, D. y Spada, M. M. (2014). Negative Recurrent ThinkingasaModerator of the Relationship Between Perceived Stress and Depressive Symptoms. Journal of Rational - Emotive \& Cognitive - Behavior Therapy, 32(4), 248-256. https://doi.org/10.1007/s10942014-0192-5

Nolen-Hoeksema,S.,Wisco,B.E.yLyubomirsky, S. (2008). Rethinking Rumination. Perspectives on Psychological Science, 3(5), 400-424. https://doi.org/10.1111/ j.1745-6924.2008.00088.x

Organización Mundial de la Salud [OMS] (2017). Día mundial de la salud mental. Autor. https://www.who.int/mental health/world-mental-health-day/2017/es/

Querstret, D. y Cropley, M. (2013). Assessing treatments used to reduce rumination and/ or worry: A systematic review. Clinical Psychology Review, 33(8), 996-1009. https://doi.org/10.1016/j.cpr.2013.08.004

*Raes, F. (2010). Rumination and worry as mediators of the relationship between selfcompassion and depression and anxiety. Personality and Individual Differences, 48(6), 757-761. https://doi.org/10.1016/j. paid.2010.01.023

*Rood, L., Roelofs, J., Bögels, S. M. y Arntz, A. (2012). The effects of experimentally induced rumination, positive reappraisal, acceptance, and distancing when thinking about a stressful event on affect states in adolescents. Journal of Abnormal Child Psychology, 40(1), 73-84. https://doi. org/10.1007/s10802-011-9544-0

*Ruscio, A. M., Gentes, E. L., Jones, J. D., Hallion, L. S., Coleman, E. S. y Swendsen, J. (2015). Rumination predicts heightened responding to stressful life events in major depressive disorder and generalized anxiety disorder. Journal of Abnormal Psychology, 124(1), 17-26. https://doi. org/10.1037/abn0000025

*Ruscio, A. M., Seitchik, A. E., Gentes, E. 
L., Jones, J. D. y Hallion, L. S. (2011). Perseverative thought: A robust predictor of response to emotional challenge in generalized anxiety disorder and major depressive disorder. Behaviour Research and Therapy, 49(12), 867-874. https:// doi.org/10.1016/j.brat.2011.10.001

*Slavish, D. C. y Graham-Engeland, J. E. (2015). Rumination mediates the relationships between depressed mood and both sleep quality and self-reported health in young adults. Journal Of Behavioral Medicine, 38(2), 204-213. https://doi.org/10.1007/ s10865-014-9595-0

Enviado: 27 de febrero de 2020

Revisado: 10 de junio 2020

Aceptado: 15 de septiembre de 2020 